The Journal of $\mathbf{N}_{\text {onlinear }} \mathbf{S}_{\text {cience and }}$ Applications

http://www.tjnsa.com

\title{
CONDITIONING OF THREE-POINT BOUNDARY VALUE PROBLEMS ASSOCIATED WITH FIRST ORDER MATRIX LYAPUNOV SYSTEMS
}

\author{
M.S.N. MURTY ${ }^{1, *}$, D. ANJANEYULU ${ }^{1}$ AND G. SURESH KUMAR ${ }^{2}$ \\ This paper is dedicated to the 60th Anniversary of Professor Themistocles M. Rassias \\ Communicated by Choonkil Park
}

\begin{abstract}
This paper deals with the study of conditioning for three-point boundary value problems associated with first order matrix Lyapunov systems, with the help of Kronecker product of matrices. Further, we obtain the close relationship between the stability bounds of the problem on one hand, and the growth behavior of the fundamental matrix solution on the other hand.
\end{abstract}

\section{INTRODUCTION}

Matrix Lyapunov type systems arise in a number of applied mathematics such as dynamical programming, optimal filters, quantum mechanics, and systems engineering. The study of conditioning of boundary value problems is an interesting area of current research due to their invaluable use in estimating the global error due to small perturbations.

In this direction, Hoog and Mattheije [1], and Murty and Lakshmi [3] have obtained results of this type for two-point boundary value problems associated with system of first order matrix differential equations satisfying two-point boundary conditions. Further, Murty and Rao [4] studied conditioning for three-point boundary value problems associated with system of first order rectangular matrix differential equations. Due to the importance of matrix Lyapunov systems

Date: Received: October 9, 2010; Revised: November 24, 2010.

*Corresponding author

(c) 2011 N.A.G.

2000 Mathematics Subject Classification. Primary 34B27, 34C10, 65F35, 65L07.

Key words and phrases. Lyapunov system; boundary value problem; Kronecker product; condition number. 
in the theory of differential equations, Murty and Rao [5] studied existence and uniqueness criteria associated with two-point boundary value problems. Further, Murty, Rao and Kumar [6] have studied controllability, observability, and realizability criteria for matrix Lyapunov systems. Furthermore, Murty and Suresh Kumar [7] obtain results on dichotomy and conditioning for two-point boundary value problems associated with matrix Lyapunov systems.

Now, we consider the general first order matrix Lyapunov system of the form

$$
L X=X^{\prime}(t)-(A(t) X(t)+X(t) B(t))=F(t), a \leq t \leq c
$$

satisfying three-point boundary conditions

$$
M X(a) S+N X(b) T+R X(c) U=Q,
$$

where $A(t), B(t), F(t) \in\left[L_{p}(a, c)\right]^{n \times n}$ for some $p$ satisfying the condition $1 \leq p<\infty$, and $M, N, R, S, T, U, Q$ are all of constant square matrices of order $n$.

In this paper we investigate the close relationship between the stability bounds of three-point boundary value problems for matrix Lyapunov systems on the one hand, and the growth behavior of the fundamental matrix solution on the other hand. We also show that condition number is the right criterion to indicate possible error amplification of the perturbed boundary conditions.

The paper is well organized as follows. In Section 2 we present some basic definitions and preliminary results relating to existence and uniqueness of solutions of the corresponding Kronecker product three-point boundary value problem associated with (1.1) satisfying (1.2). The properties of the Green's matrix are also studied. In Section 3 we discuss about conditioning of the boundary value problem and present a stability analysis of this algorithm and also show that the condition number is an important quantity in estimating the global error.

\section{Existence AND uniqueness of SOlutions}

In this section we convert the given boundary value problem into a Kronecker product three-point boundary value problem and obtain existence and uniqueness of solution of three-point boundary value problems with the help of a Green's matrix. Also study the properties of Green's matrix and obtain stability bounds with the help of Kronecker product of matrices.

Definition 2.1. [2] Let $A \in \mathbb{C}^{m \times n}\left(\mathbb{R}^{m \times n}\right)$ and $B \in \mathbb{C}^{p \times q}\left(\mathbb{R}^{p \times q}\right)$ then the Kronecker product of $A$ and $B$ written $A \otimes B$ is defined to be the partitioned matrix

$$
A \otimes B=\left[\begin{array}{cccc}
a_{11} B & a_{12} B & \ldots & a_{1 n} B \\
a_{21} B & a_{22} B & \ldots & a_{2 n} B \\
\cdot & \cdot & \ldots & \cdot \\
a_{m 1} B & a_{m 2} B & \ldots & a_{m n} B
\end{array}\right]
$$

is an $m p \times n q$ matrix, and is in $\mathbb{C}^{m p \times n q}\left(\mathbb{R}^{m p \times n q}\right)$.

Definition 2.2. [2] Let $A=\left[a_{i j}\right] \in \mathbb{C}^{m \times n}\left(\mathbb{R}^{m \times n}\right)$, we denote 


$$
\hat{A}=\operatorname{Vec} A=\left[\begin{array}{c}
A_{.1} \\
A_{.2} \\
\cdot \\
\cdot \\
A_{. n}
\end{array}\right], \text { where } A_{. j}=\left[\begin{array}{c}
a_{1 j} \\
a_{2 j} \\
\cdot \\
\cdot \\
a_{m j}
\end{array}\right](1 \leq j \leq n)
$$

The Kronecker product has the following properties and rules, we refer to [2] and[6].

1. $(A \otimes B)^{*}=A^{*} \otimes B^{*}$

2. $(A \otimes B)^{-1}=A^{-1} \otimes B^{-1}$

3. The mixed product rule;

$(A \otimes B)(C \otimes D)=(A C \otimes B D)$

this rule holds, provided the dimension of the matrices are such that the various expressions exist.

4. $\|A \otimes B\|=\|A\|\|B\|$

5. $(A+B) \otimes C=(A \otimes C)+(B \otimes C)$

6. If $A(t)$ and $B(t)$ are matrices, then

$(A \otimes B)^{\prime}=A^{\prime} \otimes B+A \otimes B^{\prime}\left({ }^{\prime}=d / d t\right)$

7. $\operatorname{Vec}(A Y B)=\left(B^{*} \otimes A\right) \operatorname{Vec} Y$

8. If $A$ and $B$ are matrices both of order $n \times n$ then

(i) $\operatorname{Vec}(A X)=\left(I_{n} \otimes A\right) \operatorname{Vec} X$

(ii) $\operatorname{Vec}(X A)=\left(A^{*} \otimes I_{n}\right) \operatorname{Vec} X$.

Now by applying the Vec operator to the matrix Lyapunov system (1.1), satisfying the boundary conditions (1.2), and using the above properties, we have

$$
\hat{X}^{\prime}(t)=H(t) \hat{X}(t)+\hat{F}(t)
$$

satisfying

$$
\left(S^{*} \otimes M\right) \hat{X}(a)+\left(T^{*} \otimes N\right) \hat{X}(b)+\left(U^{*} \otimes R\right) \hat{X}(c)=\hat{Q}
$$

where $H(t)=\left(B^{*} \otimes I_{n}\right)+\left(I_{n} \otimes A\right), \hat{X}=\operatorname{Vec} X, \hat{F}=\operatorname{Vec} F$, and $\hat{Q}=\operatorname{Vec} Q$.

The corresponding homogeneous system of (2.1) is

$$
L \hat{X}=\hat{X}^{\prime}(t)-H(t) \hat{X}(t)=0 .
$$

Lemma 2.1. Let $Y(t)$ and $Z(t)$ be the fundamental matrices for the systems

$$
X^{\prime}(t)=A(t) X(t)
$$

and

$$
X^{\prime}(t)=B^{*}(t) X(t)
$$

respectively. Then the matrix $Z(t) \otimes Y(t)$ is a fundamental matrix of $(2.3)$, and every solution of (2.3) is of the form $\hat{X}(t)=(Z(t) \otimes Y(t)) c$, where $c$ is a $n^{2}$-column vector. 
Proof. Consider

$$
\begin{aligned}
(Z(t) \otimes Y(t))^{\prime} & =\left(Z^{\prime}(t) \otimes Y(t)\right)+\left(Z(t) \otimes Y^{\prime}(t)\right) \\
& =\left(B^{*}(t) Z(t) \otimes Y(t)\right)+(Z(t) \otimes A(t) Y(t)) \\
& =\left(B^{*}(t) \otimes I_{n}\right)(Z(t) \otimes Y(t))+\left(I_{n} \otimes A(t)\right)(Z(t) \otimes Y(t)) \\
& =\left[B^{*}(t) \otimes I_{n}+I_{n} \otimes A(t)\right](Z(t) \otimes Y(t)) \\
& =H(t)(Z(t) \otimes Y(t)) .
\end{aligned}
$$

Since $Y(t)$ and $Z(t)$ are nonsingular, then $Z(t) \otimes Y(t)$ is also nonsingular. Hence $Z(t) \otimes Y(t)$ is a fundamental matrix of $(2.3)$. Clearly $\hat{X}(t)=(Z(t) \otimes Y(t)) c$, is a solution of (2.3), and every solution is of this form.

Theorem 2.1. Let $Y(t)$ and $Z(t)$ be the fundamental matrices for the systems (2.4) and (2.5), then any solution of non-homogeneous system (2.1) is of the form

$$
\hat{X}(t)=(Z(t) \otimes Y(t)) c+(Z(t) \otimes Y(t)) \int_{a}^{t}\left(Z^{-1}(s) \otimes Y^{-1}(s)\right) \hat{F}(s) d s .
$$

Proof. First, we show that any solution of (2.1) is of the form

$\hat{X}(t)=(Z(t) \otimes Y(t)) c+\widetilde{X}(t)$, where $\widetilde{X}(t)$ is a particular solution of $(2.1)$ and is given by

$$
\widetilde{X}(t)=\int_{a}^{t}(Z(t) \otimes Y(t))\left(Z^{-1}(s) \otimes Y^{-1}(s)\right) \hat{F}(s) d s .
$$

Let $u(t)$ be any other solution of $(2.1)$, write $w(t)=u(t)-\widetilde{X}(t)$, then $w(t)$ satisfies (2.3), hence $w(t)=(Z(t) \otimes Y(t)) c, u(t)=(Z(t) \otimes Y(t)) c+\widetilde{X}(t)$.

Next, we consider the vector $\widetilde{X}(t)=(Z(t) \otimes Y(t)) v(t)$, where $v(t)$ is an arbitrary vector to be determined, so as to satisfy equation (2.1). Consider

$$
\begin{aligned}
& \tilde{X}^{\prime}(t)=(Z(t) \otimes Y(t))^{\prime} v(t)+(Z(t) \otimes Y(t)) v^{\prime}(t) \\
\Rightarrow & H(t) \tilde{X}(t)+\hat{F}(t)=H(t)(Z(t) \otimes Y(t)) v(t)+(Z(t) \otimes Y(t)) v^{\prime}(t) \\
\Rightarrow & (Z(t) \otimes Y(t)) v^{\prime}(t)=\hat{F}(t) \\
\Rightarrow & v^{\prime}(t)=\left(Z^{-1}(t) \otimes Y^{-1}(t)\right) \hat{F}(t) \\
\Rightarrow & v(t)=\int_{a}^{t}\left(Z^{-1}(s) \otimes Y^{-1}(s)\right) \hat{F}(s) d s .
\end{aligned}
$$

Hence the desired expression follows immediately.

Definition 2.3. The system (2.3) satisfying

$$
\left(S^{*} \otimes M\right) \hat{X}(a)+\left(T^{*} \otimes N\right) \hat{X}(b)+\left(U^{*} \otimes R\right) \hat{X}(c)=0
$$

is called a homogeneous Kronecker product boundary value problem. By a solution of this problem we mean a solution of (2.3) whose values at ' $a$ ', ' $b$ ' and ' $c$ ' are such that the relation (2.6) is satisfied.

Definition 2.4. The dimension of the solution space of the homogeneous boundary value problem (2.3) satisfying (2.6) is called the index of compatibility of the 
problem. If the index of compatibility is zero then we say that the boundary value problem is incompatible.

Definition 2.5. If $Y(t), Z(t)$ are fundamental matrices for the systems (2.4), (2.5), then the matrix

$$
D=\left(S^{*} \otimes M\right)(Z(a) \otimes Y(a))+\left(T^{*} \otimes N\right)(Z(b) \otimes Y(b))+\left(U^{*} \otimes R\right)(Z(c) \otimes Y(c))
$$

is called the characteristic matrix for the boundary value problem (2.3) and (2.6).

Theorem 2.2. Let $Y(t), Z(t)$ be the fundamental matrices for the systems (2.4), (2.5) and suppose that the homogeneous boundary value problem (2.3) satisfying (2.6) is incompatible. Then there exists a unique solution to three-point boundary value problem (2.1) satisfying (2.2) is of the form

$$
\hat{X}(t)=(Z(t) \otimes Y(t)) D^{-1} \hat{Q}+\int_{a}^{c} G(t, s) \hat{F}(s) d s
$$

where $G$ is the Green's matrix for the homogeneous boundary value problem (2.3) and (2.6).

Proof. From Theorem 2.1 any solution of (2.1) is of the form

$$
\hat{X}(t)=(Z(t) \otimes Y(t)) C+(Z(t) \otimes Y(t)) \int_{a}^{t}\left(Z^{-1}(s) \otimes Y^{-1}(s)\right) \hat{F}(s) d s,
$$

where $(Z(t) \otimes Y(t))$ is a fundamental matrix for the equation (2.3) and $C$ is constant matrix. Substituting (2.9) in (2.6), we get

$$
\begin{gathered}
{\left[\left(S^{*} \otimes M\right)(Z(a) \otimes Y(a))\right] C+\left[\left(T^{*} \otimes N\right)(Z(b) \otimes Y(b))\right] C} \\
\left.+\left(T^{*} \otimes N\right)(Z(b) \otimes Y(b)) \int_{a}^{b}(Z(s) \otimes Y(s))^{-1} \hat{F}(s) d s+\left[\left(U^{*} \otimes R\right) Z(c) \otimes Y(c)\right)\right] C \\
+\left(U^{*} \otimes R\right)(Z(c) \otimes Y(c)) \int_{a}^{c}(Z(s) \otimes Y(s))^{-1} \hat{F}(s) d s=0 \\
\Rightarrow C=-D^{-1}\left[\left(T^{*} \otimes N\right)(Z(b) \otimes Y(b)) \int_{a}^{b}(Z(s) \otimes Y(s))^{-1} \hat{F}(s) d s\right. \\
\left.+\left(U^{*} \otimes R\right)(Z(c) \otimes Y(c)) \int_{a}^{c}(Z(s) \otimes Y(s))^{-1} \hat{F}(s) d s\right]
\end{gathered}
$$

where $D$ is the characteristic matrix for the boundary value problem. Thus

$$
\hat{X}(t)=\int_{a}^{c} G(t, s) \hat{F}(s) d s
$$


where

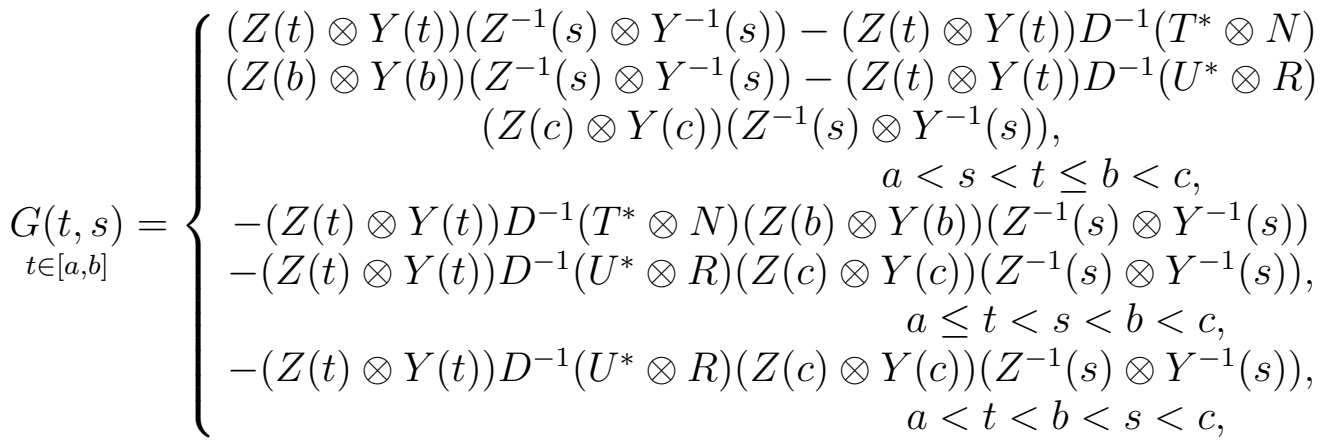

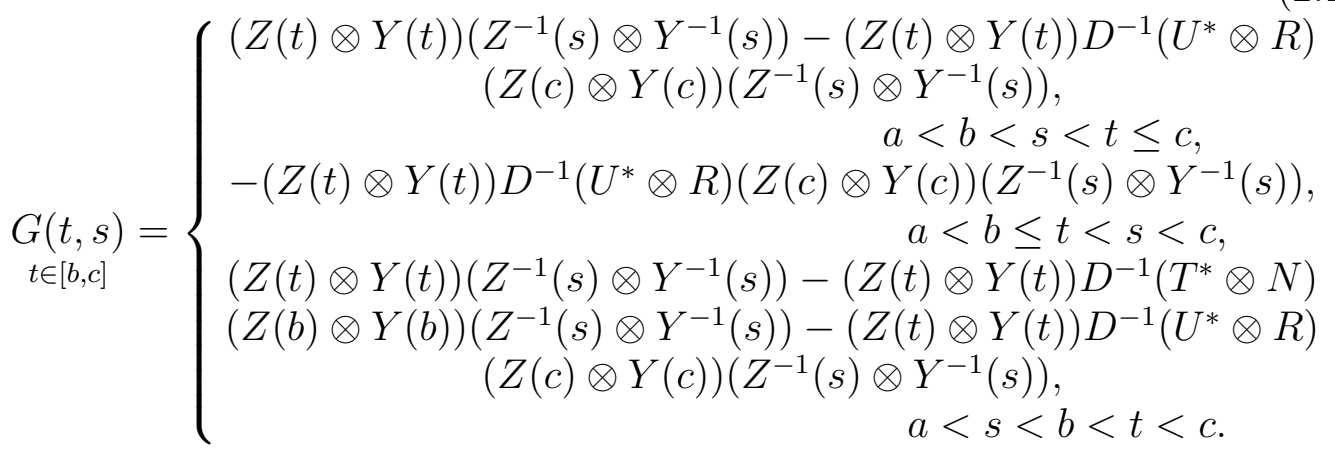

Theorem 2.3. The Green's matrix has the following properties:

(i) $G(t, s)$ as a function of $t$ with fixed $s$ have continuous derivatives everywhere except at $t=s$. At the point $t=s, G(t, s)$ has a jump discontinuity and its jump is

$$
G\left(s^{+}, s\right)-G\left(s^{-}, s\right)=I .
$$

(ii) $G(t, s)$ is a formal solution of the homogeneous boundary value problem (2.3) satisfying (2.6). G fails to be a true solution because of the discontinuity at $t=s$.

(iii) $G(t, s)$ satisfying the properties (i) and (ii) is unique.

Proof. The proof of this theorem is obvious.

We shall now see how the expression (2.8) can be used to examine the conditioning of $(2.1),(2.2)$. We make use of the following notations. Let

$$
\|v\|_{p}=\left[\int_{a}^{c}|v(s)|^{p} d s\right]^{\frac{1}{p}}, \quad 1 \leq p<\infty,
$$

and

$$
\|v\|_{\infty}=\sup _{t \in[a, c]}|v(t)|
$$

be its limiting value as $p \rightarrow \infty$. Then we have from (2.8)

$$
\|\hat{X}\|=\|\hat{X}\|_{\infty} \leq \eta|\hat{Q}|+\gamma_{q}\|\hat{F}\|_{p}, \frac{1}{p}+\frac{1}{q}=1
$$


where

$$
\eta=\left\|(Z(t) \otimes Y(t)) D^{-1}\right\|
$$

and

$$
\gamma_{q}=\sup _{t \in[a, c]}\left[\int_{a}^{c}|G(t, s)|^{q} d s\right]^{\frac{1}{q}} .
$$

The most appropriate norm in (2.12) actually depends on the problem under consideration. We shall discuss the case when $p=1$, and all the arguments used here can be extended easily to an arbitrary $p, 1<p<\infty$.

When $p=1,(2.12)-(2.14)$ reduce to

$$
\begin{gathered}
\|\hat{X}\| \leq \eta|\hat{Q}|+\gamma\|\hat{F}\|, \\
\eta=\left\|(Z(t) \otimes Y(t)) D^{-1}\right\|,
\end{gathered}
$$

and

$$
\gamma=\sup _{t, s}|G(t, s)|
$$

If in addition, we assume that the boundary conditions (2.2) are scaled in such a way that

$$
\left(S^{*} S \otimes M M^{*}\right)+\left(T^{*} T \otimes N N^{*}\right)+\left(U^{*} U \otimes R R^{*}\right)=I_{n^{2}}
$$

then

$$
\left|(Z(t) \otimes Y(t)) D^{-1}\right|^{2}=\left|G(t, a) G^{*}(t, a)+G(t, b) G^{*}(t, b)+G(t, c) G^{*}(t, c)\right|,
$$

and hence

$$
\begin{gathered}
\eta^{2} \leq \gamma^{2}+\gamma^{2}+\gamma^{2} \\
\eta \leq \sqrt{3} \gamma .
\end{gathered}
$$

Hence the stability constant $\gamma$ gives a measure for the sensitivity of (2.1) satisfying (2.2) to the changes in the data. Further, we note from (2.16), (2.17) that both the fundamental matrix, and the boundary conditions (2.2) will actually determine the magnitude of the stability constants $\eta$ and $\gamma$. Thus it is possible to construct systems for which no boundary conditions exist such that $\eta$ and $\gamma$ are of moderate size; it is also possible to find boundary conditions for (2.1) so that $\eta$ and $\gamma$ are large. Hence, if system (2.1) can support a well conditioned problem then the conditioning is intimately related to the choice of the boundary conditions.

\section{Conditioning of three-Point boundary value problems}

In this section we show that the condition number is the right criterion to indicate possible error amplification of the perturbed boundary conditions.

If the solution of the boundary value problem

$$
\hat{X}(t)=H(t) \hat{X}(t)+\hat{F}(t)
$$

satisfying

$$
\left(I_{n} \otimes M\right) \hat{X}(a)+\left(I_{n} \otimes N\right) \hat{X}(b)+\left(I_{n} \otimes R\right) \hat{X}(c)=\hat{Q}
$$


(for convenience taking $S=I_{n}, T=I_{n}$ and $U=I_{n}$ in (2.2)) is unique, then the characteristic matrix

$$
D=\left(I_{n} \otimes M\right)(Z(a) \otimes Y(a))+\left(I_{n} \otimes N\right)(Z(b) \otimes Y(b))+\left(I_{n} \otimes R\right)(Z(c) \otimes Y(c))
$$

must be non-singular, and in this case the boundary value problem is said to be well-posed.

Definition 3.1. The condition number $\eta$ of the boundary value problem (3.1), (3.2) is defined as

$$
\eta=\sup _{a \leq t \leq c}\left\|(Z(t) \otimes Y(t)) D^{-1}\right\| .
$$

It is easily seen that, the number $\eta$ is independent of the choice of the fundamental matrix.

We consider the variation $\hat{X}(t)$ of $(3.1)$ with respect to the small perturbation in the boundary conditions, the perturbation of (3.2) in the form

$$
\begin{aligned}
{\left[I_{n} \otimes(M+\right.} & \delta M)] \hat{X}(a)+\left[I_{n} \otimes(N+\delta N)\right] \hat{X}(b) \\
& +\left[I_{n} \otimes(R+\delta R)\right] \hat{X}(c)=\hat{Q}+\delta \hat{Q}
\end{aligned}
$$

Then the perturbed characteristic matrix

$$
\begin{aligned}
D_{1}=\left[I_{n} \otimes(M+\delta M)\right](Z(a) \otimes Y(a))+\left[I_{n} \otimes(N+\delta N)\right](Z(b) \otimes Y(b)) \\
\quad+\left[I_{n} \otimes(R+\delta R)\right](Z(c) \otimes Y(c)) \\
=\left[\left(I_{n} \otimes M\right)+\left(I_{n} \otimes \delta M\right)\right](Z(a) \otimes Y(a)) \\
\quad+\left[\left(I_{n} \otimes N\right)+\left(I_{n} \otimes \delta N\right)\right](Z(b) \otimes Y(b)) \\
\quad+\left[\left(I_{n} \otimes R\right)+\left(I_{n} \otimes \delta R\right)\right](Z(c) \otimes Y(c)) \\
=\left[\left(I_{n} \otimes M\right)(Z(a) \otimes Y(a))+\left(I_{n} \otimes N\right)(Z(b) \otimes Y(b))+\left(I_{n} \otimes R\right)(Z(c) \otimes Y(c))\right] \\
\quad+\left(I_{n} \otimes \delta M\right)(Z(a) \otimes Y(a))+\left(I_{n} \otimes \delta N\right)(Z(b) \otimes Y(b)) \\
\quad+\left(I_{n} \otimes \delta R\right)(Z(c) \otimes Y(c)) \\
=D+\delta D .
\end{aligned}
$$

Assume that $D_{1}$ is nonsingular. Let $\widetilde{X}(t)$ be the unique solution of (3.1) satisfying (3.4).

Lemma 3.1. $\left\|\delta D D^{-1}\right\| \leq(\|\delta M\|+\|\delta N\|+\|\delta R\|) \eta$.

Proof. Consider

$$
\begin{aligned}
\left\|\delta D D^{-1}\right\|= & \|\left[( I _ { n } \otimes \delta M ) \left(Z(a) \otimes Y(a)+\left(I_{n} \otimes \delta N\right)(Z(b) \otimes Y(b))\right.\right. \\
& \left.+\left(I_{n} \otimes \delta R\right)(Z(c) \otimes Y(c))\right] D^{-1} \| \\
\leq & \left\|\left(I_{n} \otimes \delta M\right)\right\|\left\|(Z(a) \otimes Y(a)) D^{-1}\right\|+\left\|\left(I_{n} \otimes \delta N\right)\right\|\left\|(Z(b) \otimes Y(b)) D^{-1}\right\| \\
& +\left\|\left(I_{n} \otimes \delta R\right)\right\|\left\|(Z(c) \otimes Y(c)) D^{-1}\right\| \\
= & \left\|I_{n}\right\|\|\delta M\|\left\|(Z(a) \otimes Y(a)) D^{-1}\right\|+\left\|I_{n}\right\|\|\delta N\|\left\|(Z(b) \otimes Y(b)) D^{-1}\right\| \\
& +\left\|I_{n}\right\|\|\delta R\|\left\|(Z(c) \otimes Y(c)) D^{-1}\right\| \\
\leq & (\|\delta M\|+\|\delta N\|+\|\delta R\|)\left\|(Z(t) \otimes Y(t)) D^{-1}\right\| \\
\leq & (\|\delta M\|+\|\delta N\|+\|\delta R\|) \eta .
\end{aligned}
$$

Theorem 3.1. Let $\epsilon>0$ be such that $0<\epsilon<\frac{1}{(1+k) \delta \eta}$, where

$$
\delta=\max \{\|\delta M\|,\|\delta N\|, 2\|\delta R\|,\|\delta \hat{Q}\|,\|\delta D\|\}
$$


and

$$
k=\int_{a}^{c}\left\|\left(Z^{-1}(s) \otimes Y^{-1}(s)\right) \hat{F}(s)\right\| d s .
$$

Then the solution $\widetilde{X}(t)$ of (3.1) satisfying (3.4) is such that

$$
\begin{array}{r}
\delta \eta(1-k)(\|Z(a)\|\|Y(a)\|+\|Z(b)\|\|Y(b)\|+2\|Z(c)\|\|Y(c)\|) \leq \max _{t \in[a, c]}\|\tilde{X}(t)-\hat{X}(t)\| \\
\leq \delta \eta(1+k)(\|Z(a)\|\|Y(a)\|+\|Z(b)\|\|Y(b)\|+2\|Z(c)\|\|Y(c)\|) .
\end{array}
$$

Proof. Here we consider the case when $s \in[a, b]$ and the other cases can be dealt with similarly. From Theorem 2.2 any solution of $X(t)$ of (1.1) and (1.2) is given by (2.8). And any solution $\widetilde{X}(t)$ of (3.1) satisfying (3.3) is given by

$$
\widetilde{X}(t)=(Z(t) \otimes Y(t)) D_{1}^{-1}(\hat{Q}+\delta \hat{Q})+\int_{a}^{c} G_{1}(t, s) \hat{F}(s) d s,
$$

where

$$
\begin{aligned}
& G_{1}(t, s)=\left\{\begin{array}{c}
(Z(t) \otimes Y(t))\left(Z^{-1}(s) \otimes Y^{-1}(s)\right)-(Z(t) \otimes Y(t)) D_{1}^{-1}\left(I_{n} \otimes N_{1}\right) \\
(Z(b) \otimes Y(b))\left(Z^{-1}(s) \otimes Y^{-1}(s)\right)-(Z(t) \otimes Y(t)) D_{1}^{-1}\left(I_{n} \otimes R_{1}\right) \\
(Z(c) \otimes Y(c))\left(Z^{-1}(s) \otimes Y^{-1}(s)\right), \\
a<s<t \leq b<c, \\
-(Z(t) \otimes Y(t)) D_{1}^{-1}\left(I_{n} \otimes N_{1}\right)(Z(b) \otimes Y(b))\left(Z^{-1}(s) \otimes Y^{-1}(s)\right) \\
-(Z(t) \otimes Y(t)) D_{1}^{-1}\left(I_{n} \otimes R_{1}\right)(Z(c) \otimes Y(c))\left(Z^{-1}(s) \otimes Y^{-1}(s)\right), \\
a \leq t<s<b<c, \\
-(Z(t) \otimes Y(t)) D_{1}^{-1}\left(I_{n} \otimes R_{1}\right)(Z(c) \otimes Y(c))\left(Z^{-1}(s) \otimes Y^{-1}(s)\right), \\
a<t<b<s<c,
\end{array}\right. \\
& G_{1}(t, s)=\left\{\begin{array}{c}
(Z(t) \otimes Y(t))\left(Z^{-1}(s) \otimes Y^{-1}(s)\right)-(Z(t) \otimes Y(t)) D_{1}^{-1}\left(I_{n} \otimes R_{1}\right) \\
(Z(c) \otimes Y(c))\left(Z^{-1}(s) \otimes Y^{-1}(s)\right), \\
a<b<s<t \leq c, \\
-(Z(t) \otimes Y(t)) D_{1}^{-1}\left(I_{n} \otimes R_{1}\right)(Z(c) \otimes Y(c))\left(Z^{-1}(s) \otimes Y^{-1}(s)\right), \\
a<b \leq t<s<c, \\
(Z(t) \otimes Y(t))\left(Z^{-1}(s) \otimes Y^{-1}(s)\right)-(Z(t) \otimes Y(t)) D_{1}^{-1}\left(I_{n} \otimes N_{1}\right) \\
(Z(b) \otimes Y(b))\left(Z^{-1}(s) \otimes Y^{-1}(s)\right) \\
-(Z(t) \otimes Y(t)) D_{1}^{-1}\left(I_{n} \otimes R_{1}\right)(Z(c) \otimes Y(c))\left(Z^{-1}(s) \otimes Y^{-1}(s)\right), \\
a<s<b<t<c .
\end{array}\right. \\
& M_{1}=M+\delta M, N_{1}=N+\delta N \text { and } R_{1}=R+\delta R .
\end{aligned}
$$

Now consider

$$
\begin{aligned}
&\|\tilde{X}(t)-\hat{X}(t)\| \leq \|(Z(t) \otimes Y(t))\left[D_{1}^{-1}(\hat{Q}+\delta \hat{Q})-D^{-1} \hat{Q}\right] \| \\
&+\int_{a}^{t} \|(Z(t) \otimes Y(t))\left[D_{1}^{-1}\left(I_{n} \otimes M_{1}\right)-D^{-1}\left(I_{n} \otimes M\right)\right] \\
&(Z(a) \otimes Y(a))\left(Z^{-1}(s) \otimes Y^{-1}(s)\right) \hat{F}(s) \| d s \\
&+\int_{t}^{b} \|(Z(t) \otimes Y(t))\left[D_{1}^{-1}\left(I_{n} \otimes N_{1}\right)-D^{-1}\left(I_{n} \otimes N\right)\right] \\
&(Z(b) \otimes Y(b))\left(Z^{-1}(s) \otimes Y^{-1}(s)\right) \hat{F}(s) \| d s .
\end{aligned}
$$




$$
\begin{gathered}
+\int_{t}^{b} \|(Z(t) \otimes Y(t))\left[D_{1}^{-1}\left(I_{n} \otimes R_{1}\right)-D^{-1}\left(I_{n} \otimes R\right)\right] \\
(Z(c) \otimes Y(c))\left(Z^{-1}(s) \otimes Y^{-1}(s)\right) \hat{F}(s) \| d s \\
+\int_{b}^{c} \|(Z(t) \otimes Y(t))\left[D_{1}^{-1}\left(I_{n} \otimes R_{1}\right)-D^{-1}\left(I_{n} \otimes R\right)\right] \\
(Z(c) \otimes Y(c))\left(Z^{-1}(s) \otimes Y^{-1}(s)\right) \hat{F}(s) \| d s .
\end{gathered}
$$

In accordance with the linear terms, we have the following rough estimates;

$$
\begin{aligned}
D_{1}^{-1}(\hat{Q}+\delta \hat{Q})-D^{-1} \hat{Q} & =(D+\delta D)^{-1}(\hat{Q}+\delta \hat{Q})-D^{-1} \hat{Q} \\
= & D^{-1}\left(I_{n^{2}}+D^{-1} \delta D\right)^{-1}(\hat{Q}+\delta \hat{Q})-D^{-1} \hat{Q} \\
\cong & D^{-1}\left[I_{n^{2}}-D^{-1} \delta D\right](\hat{Q}+\delta \hat{Q})-D^{-1} \hat{Q} \\
& \cong D^{-1} \delta \hat{Q} .
\end{aligned}
$$

Similarly

$$
\begin{gathered}
D_{1}^{-1}\left(I_{n} \otimes M_{1}\right)-D^{-1}\left(I_{n} \otimes M\right) \cong D^{-1}\left(I_{n} \otimes \delta M\right), \\
D_{1}^{-1}\left(I_{n} \otimes N_{1}\right)-D^{-1}\left(I_{n} \otimes N\right) \cong D^{-1}\left(I_{n} \otimes \delta N\right) .
\end{gathered}
$$

and

$$
D_{1}^{-1}\left(I_{n} \otimes R_{1}\right)-D^{-1}\left(I_{n} \otimes R\right) \cong D^{-1}\left(I_{n} \otimes \delta R\right) .
$$

Using these estimates in (3.5), we get

$$
\begin{aligned}
& \|\widetilde{X}(t)-\hat{X}(t)\| \leq\left\|(Z(t) \otimes Y(t)) D^{-1} \delta \hat{Q}\right\| \\
& \quad+\int_{a}^{t}\left\|(Z(t) \otimes Y(t)) D^{-1}\left(I_{n} \otimes \delta M\right)(Z(a) \otimes Y(a))\left(Z^{-1}(s) \otimes Y^{-1}(s)\right) \hat{F}(s)\right\| d s \\
& \quad+\int_{t}^{b}\left\|(Z(t) \otimes Y(t)) D^{-1}\left(I_{n} \otimes \delta N\right)(Z(b) \otimes Y(b))\left(Z^{-1}(s) \otimes Y^{-1}(s)\right) \hat{F}(s)\right\| d s \\
& \quad+\int_{t}^{b}\left\|(Z(t) \otimes Y(t)) D^{-1}\left(I_{n} \otimes \delta R\right)(Z(c) \otimes Y(c))\left(Z^{-1}(s) \otimes Y^{-1}(s)\right) \hat{F}(s)\right\| d s \\
& \quad+\int_{b}^{c}\left\|(Z(t) \otimes Y(t)) D^{-1}\left(I_{n} \otimes \delta R\right)(Z(c) \otimes Y(c))\left(Z^{-1}(s) \otimes Y^{-1}(s)\right) \hat{F}(s)\right\| d s \\
& \quad \leq\left\|(Z(t) \otimes Y(t)) D^{-1} \delta \hat{Q}\right\|+\|(Z(t) \otimes Y(t)) D^{-1}\left[\left(I_{n} \otimes \delta M\right)(Z(a) \otimes Y(a))\right. \\
& \left.+\left(I_{n} \otimes \delta N\right)(Z(b) \otimes Y(b))+2\left(I_{n} \otimes \delta R\right)(Z(c) \otimes Y(c))\right]\left\|\int_{a}^{c}\right\|\left(Z^{-1}(s) \otimes Y-1(s)\right) \hat{F}(s) \| d s \\
& \quad \leq\left\|(Z(t) \otimes Y(t)) D^{-1} \delta \hat{Q}\right\|+\left\|(Z(t) \otimes Y(t)) D^{-1}\right\| \\
& \|\left[\left(I_{n} \otimes \delta M\right)(Z(a) \otimes Y(a))+\left(I_{n} \otimes \delta N\right)\left(Z(b) \otimes Y(b)+2\left(I_{n} \otimes \delta R\right)(Z(c) \otimes Y(c))\right] \| k\right. \\
& \leq \delta \eta+\delta \eta k[\|Z(a)\|\|Y(a)\|+\|Z(b)\|\|Y(b)\|+2\|Z(c)\|\|Y(c)\|] \\
& \leq(1+k) \delta \eta[\|Z(a)\|\|Y(a)\|+\|Z(b)\|\|Y(b)\|+2\|Z(c)\|\|Y(c)\|] .
\end{aligned}
$$

The reverse inequality follows by noting the fact that

$$
\|\widetilde{X}(t)-\hat{X}(t)\| \geq\left\|(Z(t) \otimes Y(t)) D^{-1} \delta \hat{Q}\right\|-\int_{a}^{c}\left\|G_{1}(t, s)-G(t, s)\right\|\|\hat{F}(s)\| d s .
$$


One may choose $\eta$ such that

$$
\eta=\sup _{a \leq t \leq c}\|(Z(t) \otimes Y(t))\|\left\|D^{-1}\right\|,
$$

to obtain a more reliable quantity for $\eta$. The estimate in the above theorem depends on well-known quantities and on the value of the fundamental matrix at the boundary points.

\section{REFERENCES}

[1] F.R. De Hoog and R.M.M. Mattheije, On dichotomy and well conditioning in boundary value problem, SIAM J. Numer. Anal. 24 (1987),89-105. 1

[2] A. Graham, Kronecker Products and Matrix Calculus; With Applications, Ellis Horwood Ltd., England, 1981. 2

[3] K.N. Murty and P.V.S. Lakshmi, On dichotomy and well-conditioning in two-point boundary value problems, Applied Mathematics and Computations 38 (1990), 179-199. 1

[4] M.S.N. Murty and B.V. Appa Rao, On conditioning for three-point boundary value problems, Indian Journal of Mathematics 45 (2003), 211-221. 1

[5] M.S.N. Murty and B.V. Appa Rao, On two point boundary value problems for $\dot{X}=A X+$ $X B$, J. Ultra Scientist of Physical Sciences 16 (2004), 223-227. 1

[6] M.S.N. Murty, B.V. Appa Rao and G. Suresh Kumar, Controllability, observability and realizability of matrix Lyapunov systems, Bull. Korean. Math. Soc. 43 (2006), 149-159. 1, 2

[7] M.S.N. Murty and G. Suresh Kumar, On dichotomy and conditioning for two-point boundary value problems associated with first order matrix Lyapunov systems, J. Korean Math. Soc. 45 (2008), 1361-1378. 1

${ }^{1}$ Department of Applied Mathematics, Achrya Nagarjuna University-Nuzvid Campus, Nuzvid-521 201, Andra Prdesh, India

E-mail address: drmsn2002@gmail.com

${ }^{2}$ Department of Mathematics (FED-II), K L University, Vaddeswaram-522 502, Guntur, Andra Prdesh, India

E-mail address: gsk006@yahoo.com 